\title{
P 19 MORTALITY AND MEDICAL CARE AFTER BEREAVEMENT: A GENERAL PRACTICE COHORT STUDY
}

M. King ${ }^{1}$, M. Varagunam², I. Petersen ${ }^{2}$, L. Jones', L. Marston², I. Nareth ${ }^{1}{ }^{1}$ Mental Health Sciences Unit, 67-73 Riding House Street, London W1W 7EH; ${ }^{2}$ Research Department of Primary Care and Population Sciences, Royal Free Campus Rowland Hill Street, London NW3 2PF, UK

10.1136/bmjspcare-2012-000264.26

Introduction Bereaved spouses or partners are thought to be at increased risk of morbidity and mortality. However, there are few large prospective studies and results are inconsistent. We estimated the relative mortality, prescription of psychotropic medication and use of primary medical care services in adults whose cohabitee died of cancer.

Aims and Methods Cohort study using The Health Improvement Network (THIN) UK primary care database. Participants were (1) people aged over 40, who were registered with general practices and had been exposed to the death of a cohabitee from cancer; and (2) a comparison cohort frequency matched on five year age bands and sex who were cohabiting with a living partner. The baseline was chosen as six months before the date of the cancer death for the exposed group and a random date for the unexposed group. Incidence rate ratios (IRR) with 95\% confidence intervals (CI) were estimated using random effects Poisson regression to account for clustering within general practices and adjusting for other key variables. Results 92,129 patients were studied for a median follow up of 4 years. Cohabitees of patients who died of cancer were less likely to die of any cause (IRR $0.71,96 \%$ CI $0.68-0.74$ ) after adjustment for age, gender, number of non-psychotropic prescriptions 6 months before the cancer death/index date, use of psychotropic medication 6 months before the cancer death/index date, smoking, alcohol and area deprivation score. Exposed patients were more likely to receive a prescription for antidepressant or hypnotic medication and to attend their GP both before and after the death of the cohabitee.

Conclusion We did not confirm increased mortality after bereavement of a cohabitee. Our finding may reflect the high quality of care that partners of people dying from cancer in the UK receive from palliative care and primary care services. 


\section{Corrections}

doi:10.1136/bmjspcare-2012-000264.24corr1

Maher J, McConnel H. How many people need expert symptom assessment but are not in the last year of life? BMJ Support Palliat Care 2012;2:8. Hannah McConnell's surname was misspelt as 'McConnel' in this article. The correct spelling is 'McConnell'. 